Patricia A. Wisocki, Behavioral Approaches to Gerontology. In Hersen, M., Eisler, R. M. and Miller, P. M. (eds), Progress in Behavior Modification, Volume I6. Academic Press, 1984, I2 I-157. Richard A. Hussian, Behavioral Geriatrics. In Hersen, M., Eisler, R. M. and Miller, P. M. (eds), Progress in Behavior Modification, Volume 16. Academic Press, I 984, 159-183.

These companion review articles lean towards each other like bookends struggling to compress an increasingly substantial body of literature. Both are a mine of information and must be required reading for all concerned with the understanding of old people's behaviour.

It is unfortunate, however, that there is a good deal of overlap between these authors in terms of content, with many references in common. At the same time, and perhaps in consequence, a few important issues such as the relative failure of behaviour modifiers to be sufficiently selective in contributing to the management of incontinence, or the growing importance of behaviourally-based family therapy with supporters of the elderly, are skated over, or completely neglected.

Wisocki attempts to deal primarily with the psychological correlates of physical impairment and diseases of ageing.

She offers a particularly helpful table (pp. 127-1 29) which under 12 main headings aligns physical changes in ageing and their possible behavioural consequences; and her coverage of the behavioural medicine of old age (pp. 137-I 1 ) is a model of clear thinking which has much to offer for the growing number of psychologists working closely with community health teams.

Wisocki's review of modification of age-related clinical problems

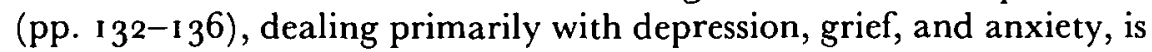
less satisfying, seeming out of place in this article and would more appropriately have been dealt with by Hussian. This is not to say that such problems do not occur frequently in conjunction with physical impairment and disease, but that Wisocki somehow fails to make such connections clear enough to justify her inclusion of these topics in this article.

Attempts to improve cognitive functioning are discussed in separate sections (pp. $130-13^{1}$ and pp. $141^{1-145}$ ), in which the complex issues of language retraining, visuospatial training, memory retraining and restimulation programming have to be covered at breakneck speed.

Her handling of the important subject of environmental influence on behaviour (pp. $145^{-1} 4^{8}$ ), although similarly condensed, is much more sure. 
Hussian offers a concise overview of the current status of application of behavioural methodology to the treatment of inappropriate behaviour by the elderly. He summarises our understanding of treatment of behavioural deficits and excesses (pp. I61-167) with remarkable facility, and proceeds to an original discussion (pp. $167-170$ ) of antecedent control - manipulation of events preceding and possibly triggering behaviour - consistent with his reputation as one of the most provocative thinkers in this field, ever ready to reach onwards, upwards (and sometimes even over the top).

His comments on the importance of observation (pp. I 70-172) are much more down to earth, and link well with his extremely interesting conclusions on future applications of behavioural techniques (pp. 1 72-1 77). In selecting disorders of communication and of movement as research growth areas, he has pinpointed concern felt by many workers with the elderly that these are indeed topics of priority for investigation. His clinical priorities-understanding of stimulusresponse parameters, generalisation and maintenance, and improved training programmes - would also appear to be highly appropriate.

Although both Wisocki and Hussian have their lapses, their achievement is a considerable one, likely to be warmly welcomed and cited for years to come.

Oxford District Health Authority

\section{Health Care Research}

John Bond

Mary E. Charlson and Ralph I. Horwitz, 'Applying results of randomised trials to clinical practice: impact of losses before randomisation'. British Medical Journal, 289 ( (1984), 1 281-1 284 .

Many writers emphasise the importance of experimental designs, in which the essential feature is randomisation, for use in the evaluation of health care programmes. One particular form of experimental design is the randomised controlled trial, which is widely used in the evaluation of clinical practice. This article describes a survey of randomised controlled trials which focused on the generalisability of the results of each trial to clinical practice; in particular it describes the impact of losses to the studies before randomisation.

The survey used a I 2 -item questionnaire which was sent to investigators responsible for trials listed in the 1979 inventory of clinical trials compiled by the National Institute of Health. Only randomised 